Meta

Journal des tradlucteurs

Translators' Journal

\title{
Présentation
}

\section{François Peraldi}

Volume 27, numéro 1, mars 1982

Psychanalyse et traduction

URI : https://id.erudit.org/iderudit/003818ar

DOI : https://doi.org/10.7202/003818ar

Aller au sommaire du numéro

\section{Éditeur(s)}

Les Presses de l'Université de Montréal

\section{ISSN}

0026-0452 (imprimé)

1492-1421 (numérique)

Découvrir la revue

Citer ce document

Peraldi, F. (1982). Présentation. Meta, 27(1), 26-28.

https://doi.org/10.7202/003818ar

Ce document est protégé par la loi sur le droit d'auteur. L'utilisation des services d'Érudit (y compris la reproduction) est assujettie à sa politique d'utilisation que vous pouvez consulter en ligne.

https://apropos.erudit.org/fr/usagers/politique-dutilisation/
Cet article est diffusé et préservé par Érudit.

Érudit est un consortium interuniversitaire sans but lucratif composé de l’Université de Montréal, l'Université Laval et l'Université du Québec à Montréal. Il a pour mission la promotion et la valorisation de la recherche. https://www.erudit.org/fr/ 


\section{1 \\ LA TRADUCTION DU TEXTE PSYCHANALYTIQUE}

\section{PRÉSENTATION}

Dans ce chapitre, le plus conforme aux intérêts immédiats et pratiques des traducteurs, il s'est agi d'aborder les difficultés inhérentes à la traduction du texte psychanalytique fondamental : le texte de Freud.

La difficulté ne tient pas à la langue de Freud: un allemand tout à fait classique et - de l'avis de tous - d'une excellente tenue litterraire, tout juste émaillé de quelques-unes de ces tournures propres à l'usage qu'on en faisait à Vienne dans les milieux cultivés de la fin du XIX ${ }^{e}$ siècle. Elle tient bien plutôt au fait que pour dire des choses jusque-là inouies, pour exposer sa «science » nouvelle, la psychanalyse, Freud avait le choix entre surcharger son discours de néologismes et de le rendre, de ce fait, tout à fait incompréhensible — un peu comme ce qui s'est passé pour l'un des plus grands penseurs nord-américains : Charles Sanders Peirce - ou, en tout cas, accessible aux seuls spécialistes; ou bien il lui fallait prendre le risque de dire, de proposer ces conceptions entièrement nouvelles avec des concepts, voire des notions courantes, déjà usés et utilisés ailleurs, dans d'autres domaines du savoir scientifique et philosophique tout à fait étrangers à la psychanalyse, mais néanmoins bien connus de Frend et de tout lecteur cultivé de son époque, mais il prenait alors le risque d'entretenir une certaine ambiguïté sémantique, de provoquer un malentendu certain.

Ce fut pourtant ce dernier choix qui prévalut. Un choix qui ne nous semble d'ailleurs pas entièrement volontaire puisque nous avons montré que Freud a découvert la psychanalyse comme malgré lui, alors qu'il cherchait à élaborer quelque chose de beaucoup plus conforme aux idéaux scientifiques de son temps: à savoir cette psychologie mécaniciste et scientifique à la Helmholtz, qui ne cessa de le hanter. D'où la nécessité, pour Freud, de revenir sans cesse sur les concepts qui, en quelque sorte, surgissaient sous ses yeux de son propre discours, à sa plus grande surprise, d'ailleurs sans cesse réitérée et qui se manifeste à tout moment par des remarques incidentes qui émaillent son texte comme: «si l'on poursuit ce raisonnement jusqu'au bout, on est contraint d'admettre que...», même si ce qu'il lui fallait admettre, comme par exemple le fait que le trauma originel d'une hystérie puisse ne pas être lié à une séduc- 
tion réelle, allait à l'encontre de ce qu'il cherchait à construire; ou bien : «ceci n'est qu'une spéculation... mais toutefois...»; ou bien encore cette admirable anticipation de l'inconscient dans le dernier chapitre des Études sur l'hystérie :

Tous les résultats de ce procédé [il s'agit du procédé de pression des mains sur le front du patient pour favoriser le retour des souvenirs refoulés que Freud tente de substituer à l'hypnose] donnent l'impression trompeuse qu'il existe, en dehors du conscient des sujets, une intelligence supérieure qui détient et groupe dans un but déterminé d'importants matériaux psychiques. Elle semble avoir trouvé, pour le retour dans le conscient de ceux-ci, un ingénieux arrangement. Mais je suppose que cette seconde intelligence inconsciente n'est qu'apparente.

Elle l'est si peu qu'elle ne tardera pas à submerger Freud dè sa présence et à le contraindre au travail d'auto-analyse qui lui permettra de la mettre en évidence et qui s'amorcera cette même année (1895), quelques mois plus tard, avec la première analyse complète d'un rêve : le rêve de l'injection faite à Irma, pour ne se terminer qu'avec sa vie près d'un demi-siècle plus tard.

C'est suffisamment indiquer que ces concepts qui émergent l'un après l'autre: le refoulement, le refoulé, les défenses, l'inconscient, les pulsions etc. ne tirent toute leur valeur, leur pleine extension et leur spécificité, que du contexte dans lequel Freud les amène au jour, les fait surgir dans le mouvement de sa pensée, et absolument pas du sens que ces mêmes mots pouvaient avoir chez d'autres auteurs comme, par exemple, le refoulé chez Herbart, l'inconscient chez Hartman ou encore les principes économiques et dynamiques chez Helmholtz. C'est pourquoi la tentative de Henri Ellenberger de ramener les concepts freudiens aux termes apparemment semblables que l'on trouve chez les auteurs qui constituent le contexte des origines est, épistémologiquement parlant, inacceptable, pour ne pas dire profondément malhonnête.

D'où, par ailleurs, la nécessité d'une traduction particulièrement attentive aux nuances syntaxiques et syntagmatiques de la structure et du mouvement argumentatifs, au moins autant, si ce n'est plus, qu'à la pertinence philologique du choix de tel signifiant dans la langue d'arrivée pour tel signifié de la langue de départ. Je pense précisément à l'exemple par lequel j'ai choisi d'ouvrir ce chapitre: la traduction en français de l'opposition Trieb/Instinkt par pulsion/ instinct, ici discutée quant à sa pertinence par Marthe Robert d'une part et Jean Laplanche et Jean-Bertrand Pontalis de l'autre.

Rappelons tout d'abord qu'aucune traduction du texte freudien (ni d'aucun texte quel qu'il soit) n'est pensable sans une lecture préalable, une re-lecture critique, au sens structuraliste du terme, du texte freudien dans son ensemble. Rappelons au passage que Pound considérait fort justement la traduction comme l'une des cinq formes de la critique textuelle. Par re-lecture, nous entendons l'évaluation de la valeur (au sens que Saussure a donné à ce terme) des conceptsclés en fonction de l'évolution des structures générales de la pensée freudienne dans ce qu'elle a de plus innovateur.

Dans ce sens, quoi qu'en pense Marthe Robert, pourtant admirable lectricetraductrice-critique de Kafka, ce n'est absolument pas à la seule philologie qu'il convient de s'en remettre pour trancher quant au choix du terme de «pulsion» 
pour traduire en français l'allemand Trieb et pour en dégager le sens par rapport au terme d'instinct (Instinkt). Sur ce point, il nous semble que Laplanche et Pontalis élèvent les objections qui conviennent, quel que soit, par ailleurs, l'acharnement avec lequel tous les trois se retrouvent pour faire chorus dans leur dénigrement de Lacan en lui faisant dire ce qu'il n'a jamais dit de manière aussi sommaire. Laplanche et Pontalis devraient d'ailleurs d'autant mieux le savoir que c'est chez Lacan qu'ils ont lu Freud à livre ouvert. Lacan n'a jamais prétendu qu'on ne trouve pas le mot instinct chez Freud, il n'a pas cessé, par contre, de souligner que ce qui est proprement psychanalytique dans le texte freudien concerne, et ne concerne que les pulsions (Triebe) et que ces pulsions n'ont rien à voir avec les instincts des biologistes, fussent-ils grégaires.

On verra d'ailleurs dans la suite de ce chapitre, avec quel souci du détail, qui nous rappelle la remarque de Paul Valéry qu'il n'y a pas de détail dans l'exécution, des auteurs rigoureusement formés à la lecture du texte freudien par Lacan ont su entreprendre la traduction d'un texte d'une densité aussi redoutable et d'une importance aussi nodale dans l'œuvre de Freud, que Die Verneinung.

On aimerait que des manifestations aussi heureuses du «désir de traduire » réussissent à l'emporter sur les querelles boutiquières qui privent le lecteur francophone d'une traduction décente des auvres complètes de Freud. 